\title{
Identification of Structural Damage in Bridges Using High-Frequency Vibrational Responses
}

\author{
Ivana Mekjavić \\ Faculty of Civil Engineering, University of Zagreb, Kačićeva 26, 10000 Zagreb, Croatia \\ Correspondence should be addressed to Ivana Mekjavić; ivanam@grad.hr
}

Received 28 November 2014; Revised 30 March 2015; Accepted 1 April 2015

Academic Editor: Maosen Cao

Copyright ( 2015 Ivana Mekjavić. This is an open access article distributed under the Creative Commons Attribution License, which permits unrestricted use, distribution, and reproduction in any medium, provided the original work is properly cited.

\begin{abstract}
The present research aims to develop an effective and applicable structural damage detection method. A damage identification approach using only the changes of measured natural frequencies is presented. The structural damage model is assumed to be associated with a reduction of a contribution to the element stiffness matrix equivalent to a scalar reduction of the material modulus. The computational technique used to identify the damage from the measured data is described. The performance of the proposed technique on numerically simulated real concrete girder bridge is evaluated using imposed damage scenarios. To demonstrate the applicability of the proposed method by employing experimental measured natural frequencies this technique is applied for the first time to a simply supported reinforced concrete beam statically loaded incrementally to failure. The results of the damage identification procedure show that the proposed method can accurately locate the damage and predict the extent of the damage using high-frequency (here beyond the 4th order) vibrational responses.
\end{abstract}

\section{Introduction}

Damage or fault detection, as determined by changes in the dynamic properties or response of structures, is a subject that has received considerable attention in the literature. Since the changes in the stiffness of the structure, whether local or distributed, will cause changes in the modal parameters (notably natural frequencies, mode shapes, etc.), the location and the severity of damage in structure can be determined by changes in the modal characteristics [1-5]. Furthermore, since the natural frequencies are rather easy to measure with a relatively high level of accuracy, the methods based on the measurements of natural frequencies are potentially attractive [6-10]. For applications to large civil engineering structures the somewhat low sensitivity of frequency shifts to damage requires either very precise measurements of frequency change or large levels of damage. An exception to this limitation occurs at higher modal frequencies, where the modes are associated with local responses. However, over recent decades, the practical limitations involved with the excitation and identification of the resonant frequencies associated with these local modes, caused in part by high modal density and low participation factors, made them difficult to identify [11]. Raghavendrachar and Aktan [12] performed impact tests on a three-span reinforced concrete bridge with a goal of detecting local or obscure damage, as opposed to severe, global damage. The authors concluded that modal parameters may not be reliable as damage indicators if only the first few modes are measured. For this type of damage, modal information for higher modes would be required. Farrar and Cone [13] presented the results of damage-detection experiment performed on the I-40 bridge over the Rio Grande river. They identify the modal properties from the ambient test, when the bridge was undamaged, and from the forced-excitation tests for each of the damage cases. The results indicate that modal frequencies, modal damping ratios, and mode shapes may not be sensitive enough indicators to detect damage at an early enough stage to be practical. The destructive tests performed on the I- 40 bridge highlight the fact that damage typically is a local phenomenon. Local response is captured by higher frequency modes whereas lower frequency modes tend to capture the global response 
of the structure and are less sensitive to local changes in a structure [11]. Consequently, the low-frequency vibrational responses are insensitive to small damage and moreover, small damage is more easily accommodated by higher-frequency vibrational responses. Currently, new advanced instrumentation typified by the scanning laser vibrometer (SLV) preserves a high level of accuracy in high-frequency vibration measurements providing the detection of small levels of damage [14].

Ideally, a robust damage identification method should be able to identify that damage has occurred at a very early stage, locate the damage within sensor resolution being used, provide some estimate of the severity of the damage, and predict the remaining useful life of the structure. Several frequency-change sensitivity analysis methods presented in [6-8] can be used to detect and locate damage in structures; however they cannot correctly quantify damage in general cases. In order to avoid the insufficiency of the first-order sensitivity analysis neglecting second-order terms, Bicanic and Chen [9] presented a novel perturbation-based approach using the exact relationship between the changes of structural parameters and the changes of modal parameters.

Here, based on the nonlinear perturbation theory, an efficient iterative computational procedure is presented in order to identify damage in framed structures for which highorder (beyond the 4th order) measured natural frequencies are available. The efficiency of the proposed technique is evaluated through an example of the real concrete girder bridge with simulated damage and through laboratory testing of a simply supported reinforced concrete beam subjected to various levels of static load.

\section{Direct Iteration Technique}

The computational procedure for the direct iteration technique has been developed to solve the element scalar damage parameters $\alpha_{j}$ as well as the mode participation factors $C_{i k}$ [9]. The procedure consists of calculating the damage parameters, for example, crack location, from the frequency changes.

The iterative solution procedure is described in the following section. Depending on the number of available natural frequencies NF (number of equations) and the number of structural damage parameters NXE (number of unknowns), the eigenmode-stiffness sensitivity matrix $S_{i j}$ may not be square. When the number of the measured natural frequencies for the damaged structure NF is much fewer than the number of structural damage parameters NXE (finite-elements) (NF $<\mathrm{NXE}$ ), the system of equations is significantly underdetermined and the pseudoinverse solution can become ill-conditioned. In order to find a solution for what is in general an ill-conditioned system, the singular value decomposition (SVD) technique [15] is applied.

A FORTRAN computer program for structural damage identification has been developed based on the knowledge of the computational procedure presented next [16].

\subsection{Computational Procedure}

Step 1. Assume the initial mode participation factors $C_{i k}^{0}$ to be zero, that is, no changes in eigenvectors. Establish the initial values for $\alpha_{j}^{1}$ and $C_{i k}^{1}$ from

$$
\sum_{j=1}^{\mathrm{NXE}} S_{i j}^{1} \alpha_{j}^{1}=z_{i},
$$

where $S_{i j}$ and $z_{i}$ are the eigenmode-stiffness sensitivity matrix and vector, respectively, which are defined as

$$
\begin{aligned}
S_{i j}^{1} & =a_{i j i}, \\
z_{i} & =\Delta \lambda_{i}, \\
C_{i k}^{1} & =\frac{b_{k i}^{1}}{\lambda_{i}{ }^{*}-\lambda_{k}-b_{k k}^{1}},
\end{aligned}
$$

where $b_{k k}, b_{k i}$, and $b_{k l}$ can be defined in general form as

$$
b_{k i}^{1}=\sum_{j=1}^{\mathrm{NXE}} a_{k j i} \alpha_{j}^{1}
$$

and $a_{i j i}, a_{i j l}$, and $a_{k j i}$ are the eigenmode-stiffness sensitivity coefficients, which can be defined in a general form as

$$
a_{k j i}=\phi_{k}^{T} \mathbf{K}_{j} \phi_{i}
$$

where $\phi_{i}$ is the ith original eigenvector, $\mathbf{K}_{j}$ is the contribution of the $j$ th element to the global stiffness matrix, $\Delta \lambda_{i}$ is the change in the $i$ th eigenvalue, $\lambda_{k}$ is the $k$ th original eigenvalue, and a superscript $*$ refers to the damaged structure.

Step 2. Evaluate current estimate for $\alpha_{j}^{n}$ from

$$
\sum_{j=1}^{\mathrm{NXE}} S_{i j}^{n} \alpha_{j}^{n}=z_{i},
$$

where

$$
S_{i j}^{n}=a_{i j i}+\sum_{l=1, l \neq i}^{\mathrm{NM}} C_{i l}^{n-1} a_{i j l} .
$$

Step 3. Evaluate new modal participation factors $C_{i k}^{n}$ from

$$
C_{i k}^{n}=\frac{b_{k i}^{n}+\sum_{l=1, l \neq i, k}^{\mathrm{NM}} C_{i l}^{n-1} b_{k l}^{n}}{\lambda_{i}{ }^{*}-\lambda_{k}-b_{k k}^{n}},
$$

where

$$
b_{k i}^{n}=\sum_{j=1}^{\mathrm{NXE}} a_{k j i} \alpha_{j}^{n}
$$

and return to Step 2 if solution has not converged.

Once the mode participation factor $C_{i k}$ is found, the eigenvectors for the damaged structure can be calculated as

$$
\phi_{i}{ }^{*}=\phi_{i}+\sum_{k=1, k \neq i}^{\mathrm{NM}} C_{i k} \phi_{k},
$$




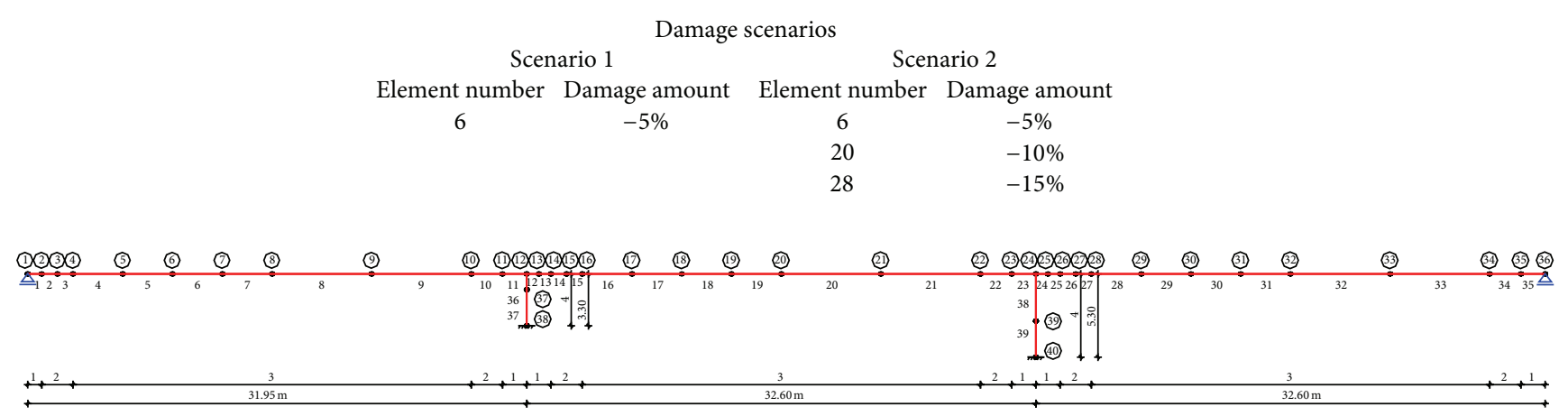

FIGURE 1: Concrete girder bridge model problem.

where the pairing of the eigenvalues for the original structure and the damaged structure can be checked using the MAC factors (Modal Assurance Criterion) [17], defined as

$$
\operatorname{MAC}(k, i)=\frac{\left|\phi_{k}{ }^{T} \phi_{i}{ }^{*}\right|^{2}}{\left|\phi_{k}{ }^{T} \phi_{k}\right|\left|\phi_{i}{ }^{* T} \phi_{i}{ }^{*}\right|} .
$$

The highest $\operatorname{MAC}(k, i)$ factors indicate the most possible pairings of the original mode $k$ and the damaged mode $i$.

\section{Numerical Example}

A model of the real concrete girder bridge comprising 39 elements, 40 nodes, and 116 degrees of freedom (DOFs), shown in Figure 1, is used to investigate the effect of the number of original eigenvectors available and the natural frequencies of the damaged structure adopted in the calculation.

It is interesting to note that, since the structure discussed here is almost symmetric, convergence difficulties may arise. From (5), it can be shown that if a structure is symmetric, the set of governing equations (1) becomes singular, and the identification process cannot proceed. If a structure is "almost" symmetric, the governing equations (1) are illconditioned, and in such a case the information of a larger number of natural frequencies for the damaged structure (greater than NXE) is required to correctly determine the location and amount of structural damage. Due to illconditioned governing equations (1), damage parameters $\alpha_{j}$ converge very slowly, leading to convergence difficulties in some cases. In order to avoid such difficult cases, some methods may be used to render the structure nonsymmetric, such as nonsymmetric element mesh generated, suitable boundary conditions selected, and additional concentrated mass applied. Consequently, the proposed method is also applicable for symmetric or near symmetric structures and a smaller number of natural frequencies for the damaged structure are required to determine structural damage [9].

In order to avoid problems associated with structural symmetry, a nonsymmetric element mesh is generated, as shown in Figure 1. Since the bridge superstructure is simply supported, the vertical and horizontal displacement at the contact point between beams and column are constrained to be the same for both the beams and column, while the
TABLE 1: Main data of the concrete girder bridge.

\begin{tabular}{lccc}
\hline Member & $\begin{array}{c}\text { Number of cross } \\
\text { sections }\end{array}$ & Area $\left(\mathrm{m}^{2}\right)$ & $\begin{array}{c}\text { Moment of inertia } \\
\left(\mathrm{m}^{4}\right)\end{array}$ \\
\hline Beam at support & 1 & 8.605 & 3.312 \\
Beam at support & 2 & 7.477 & 3.025 \\
Beam in span & 3 & 6.245 & 2.750 \\
Pier & 4 & 3.140 & 0.785 \\
\hline
\end{tabular}

rotations are free. The cross-sectional properties of each component are listed in Table 1. All structural members have the same material properties with $E=3.4 \times 10^{7} \mathrm{kN} / \mathrm{m}^{2}$ and $\rho=2.5 \mathrm{t} / \mathrm{m}^{3}$. The geometry of the structure, element numbering, and hypothetical damage scenarios are shown in Figure 1.

Two hypothetical damage scenarios are induced by reducing Young's modulus of different elements, with different magnitudes, as summarized in Figure 1. Scenario 1 simulates $5 \%$ damage in element 6 . Scenario 2 has three damaged elements. Element 6 has 5\% damage, element 20 has 10\% damage, and element 28 has $15 \%$ damage.

In most cases, the effects of damage on structural stiffness can be represented by locally reducing Young's modulus. In this study, a scalar damage model is assumed to be based on the above consideration; that is, the change of structural stiffness matrix can be expressed as the summation of changes proportional to the element matrices in the form $\Delta \mathbf{K}=\sum_{j=1}^{\mathrm{NXE}} \alpha_{j} \mathbf{K}_{j}$. This formulation makes possible the interrelation of the local decrease in stiffness to the decreases in the eigenvalues while it preserves the connectivity and symmetry of the global stiffness matrix as presented in previous researches [6-10]. The result is a set of simultaneous equations (1) that relate the changes in the eigenvalues to those of the element stiffness.

The first 35 natural frequencies for the undamaged and damaged structures of the bridge for each of the damage scenarios determined from the finite-element dynamic analyses are listed in Table 2. This table shows the differences in natural frequencies for the undamaged and damaged structures, that is, effects of damage on the natural frequencies of the first 35 modes. At mode 1 (scenario 2), the minimum change is $\Delta f=-0.01 \mathrm{~Hz}$ which represents a reduction of $0.3 \%$ (Table 2). All of the 35 modes show a reduction of their 
TABLE 2: First 35 natural frequencies for the undamaged and damaged structures.

\begin{tabular}{|c|c|c|c|c|c|c|c|}
\hline \multirow{3}{*}{ Mode } & \multirow{2}{*}{\multicolumn{3}{|c|}{$\begin{array}{l}\text { Natural frequency } \\
\qquad(\mathrm{Hz})\end{array}$}} & \multicolumn{4}{|c|}{ Difference in frequency with respect to undamaged structure } \\
\hline & & & & \multicolumn{2}{|c|}{ Scenario 1} & \multicolumn{2}{|c|}{ Scenario 2} \\
\hline & $\begin{array}{l}\text { Undamaged } \\
\text { structure }\end{array}$ & Scenario 1 & Scenario 2 & $(\mathrm{~Hz})$ & $(\%)$ & $(\mathrm{Hz})$ & $(\%)$ \\
\hline 1 & 2.996 & 2.996 & 2.987 & 0.00 & 0.00 & -0.01 & -0.30 \\
\hline 2 & 3.606 & 3.606 & 3.539 & 0.00 & 0.00 & -0.07 & -1.86 \\
\hline 3 & 3.760 & 3.745 & 3.745 & -0.01 & -0.40 & -0.01 & -0.40 \\
\hline 4 & 6.239 & 6.239 & 6.235 & 0.00 & 0.00 & -0.01 & -0.06 \\
\hline 5 & 11.928 & 11.928 & 11.813 & 0.00 & 0.00 & -0.12 & -0.96 \\
\hline 6 & 14.293 & 14.292 & 14.186 & 0.00 & 0.00 & -0.11 & -0.75 \\
\hline 7 & 14.980 & 14.926 & 14.926 & -0.05 & -0.36 & -0.05 & -0.36 \\
\hline 8 & 18.934 & 18.930 & 18.742 & 0.00 & -0.02 & -0.19 & -1.01 \\
\hline 9 & 26.643 & 26.643 & 26.281 & 0.00 & 0.00 & -0.36 & -1.36 \\
\hline 10 & 31.648 & 31.648 & 31.380 & 0.00 & 0.00 & -0.27 & -0.85 \\
\hline 11 & 33.561 & 33.548 & 33.540 & -0.01 & -0.04 & -0.02 & -0.06 \\
\hline 12 & 36.746 & 36.720 & 36.530 & -0.03 & -0.07 & -0.22 & -0.59 \\
\hline 13 & 46.699 & 46.699 & 46.208 & 0.00 & 0.00 & -0.49 & -1.05 \\
\hline 14 & 53.023 & 52.955 & 52.584 & -0.07 & -0.13 & -0.44 & -0.83 \\
\hline 15 & 54.670 & 54.660 & 54.112 & -0.01 & -0.02 & -0.56 & -1.02 \\
\hline 16 & 59.232 & 59.035 & 58.996 & -0.20 & -0.33 & -0.24 & -0.40 \\
\hline 17 & 70.642 & 70.640 & 70.375 & 0.00 & 0.00 & -0.27 & -0.38 \\
\hline 18 & 74.713 & 74.608 & 74.365 & -0.10 & -0.14 & -0.35 & -0.47 \\
\hline 19 & 81.175 & 81.150 & 80.450 & -0.02 & -0.03 & -0.72 & -0.89 \\
\hline 20 & 90.023 & 89.795 & 89.653 & -0.23 & -0.25 & -0.37 & -0.41 \\
\hline 21 & 92.564 & 92.424 & 91.303 & -0.14 & -0.15 & -1.26 & -1.36 \\
\hline 22 & 97.630 & 97.597 & 97.067 & -0.03 & -0.03 & -0.56 & -0.58 \\
\hline 23 & 108.580 & 108.386 & 108.211 & -0.19 & -0.18 & -0.37 & -0.34 \\
\hline 24 & 114.964 & 114.942 & 114.221 & -0.02 & -0.02 & -0.74 & -0.65 \\
\hline 25 & 124.176 & 124.142 & 123.241 & -0.03 & -0.03 & -0.94 & -0.75 \\
\hline 26 & 133.840 & 133.800 & 132.782 & -0.04 & -0.03 & -1.06 & -0.79 \\
\hline 27 & 140.715 & 140.578 & 140.040 & -0.14 & -0.10 & -0.68 & -0.48 \\
\hline 28 & 150.078 & 150.064 & 149.134 & -0.01 & -0.01 & -0.94 & -0.63 \\
\hline 29 & 157.242 & 157.093 & 156.630 & -0.15 & -0.09 & -0.61 & -0.39 \\
\hline 30 & 164.279 & 164.279 & 163.880 & 0.00 & 0.00 & -0.40 & -0.24 \\
\hline 31 & 165.131 & 165.090 & 164.276 & -0.04 & -0.02 & -0.85 & -0.52 \\
\hline 32 & 173.047 & 173.043 & 172.217 & 0.00 & 0.00 & -0.83 & -0.48 \\
\hline 33 & 196.072 & 195.862 & 194.716 & -0.21 & -0.11 & -1.36 & -0.69 \\
\hline 34 & 199.703 & 199.646 & 198.997 & -0.06 & -0.03 & -0.71 & -0.35 \\
\hline 35 & 211.179 & 210.875 & 209.775 & -0.30 & -0.14 & -1.40 & -0.66 \\
\hline
\end{tabular}

natural frequencies. These differences can be estimated with precision of $\Delta f< \pm 0.01 \mathrm{~Hz}$ in real modal tests as it is presented in $[18,19]$. Furthermore, because during the modal tests performed in $[18,19]$ the temperature was almost stationary, the observed changes in the natural frequencies cannot be related to temperature changes. However, due to practical testing limitations, only the natural frequencies of the first three bending modes could be tracked reliably under the test conditions in [18].

From a testing standpoint it is more difficult to excite the higher frequency response of a structure as more energy is required to produce measurable response at these higher frequencies than at the lower frequencies [11]. These factors contribute to only limited applications of vibration-based damage detection technology to large civil engineering structures.

The pairings of the original eigenvectors and the modified eigenvectors are assured by using MAC values as defined in (11), resulting in diagonal values close to unit (higher than 0.997). This implies that the modes for the damaged structure obtained from the direct iteration technique match very well the corresponding modes for the original structure.

In order to study the effectiveness of the proposed direct iteration technique with respect to the required amount of modal information, various numbers of original eigenvectors and natural frequencies for the damaged structure are 


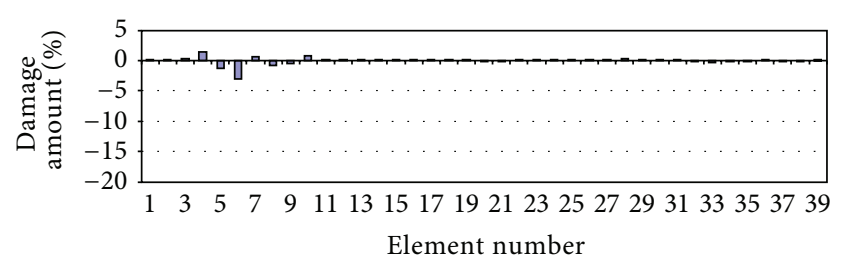

(a)

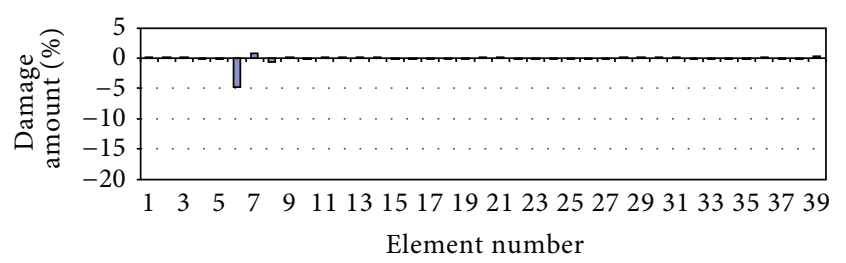

(b)

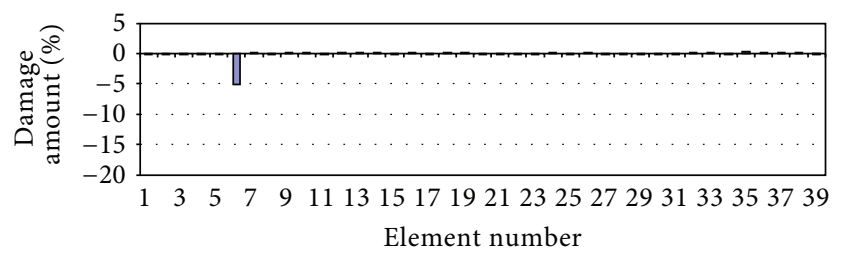

(c)

FIGURE 2: Inverse damage predictions for scenario 1 affected by the number of damaged-structure frequencies (NF), 60 original eigenvectors (NM) used, (a) 13 damaged-structure frequencies used, (b) 20 damaged-structure frequencies used, and (c) 35 damagedstructure frequencies used.

selected for the identification process for damage scenarios 1-2, as shown in Figures 2 and 3.

From the results, it can be seen that only limited knowledge of the original eigenvectors is required, even 60 original eigenvectors (half the number of all original eigenvectors) are sufficient to correctly predict the structural damage, which makes the proposed approach applicable to large scale structures. For a case with small damage (scenario 1) both the location and the amount of damage can be correctly estimated using only 13 natural frequencies for the damaged structure. For a case with more serious damage (scenario 2) structural damage can be roughly predicted using 20 natural frequencies for the damaged structure. As expected, the predictions of structural damage improve with an increase of the number of damaged-structure frequencies used, reaching the values very close to the exact solution when 35 damagedstructure frequencies are used.

\section{Experimental Study on a Beam Model}

4.1. Experimental Setup. To demonstrate the effectiveness of the presented damage identification method, a simply supported reinforced concrete beam was used as test structure. The beam was $3 \mathrm{~m}$ long by $20 \mathrm{~cm}$ wide by $30 \mathrm{~cm}$ deep. A sketch of the structure tested in the lab and the experimental setup is shown in Figure 4.

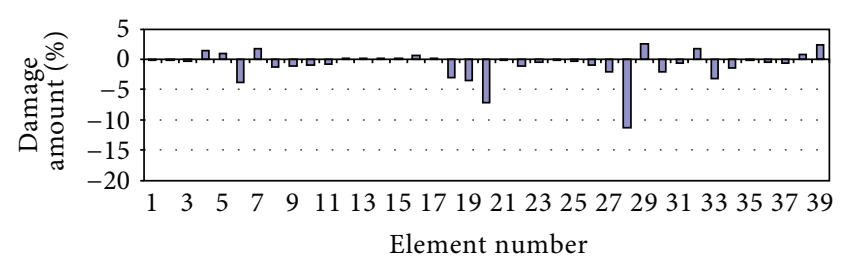

(a)

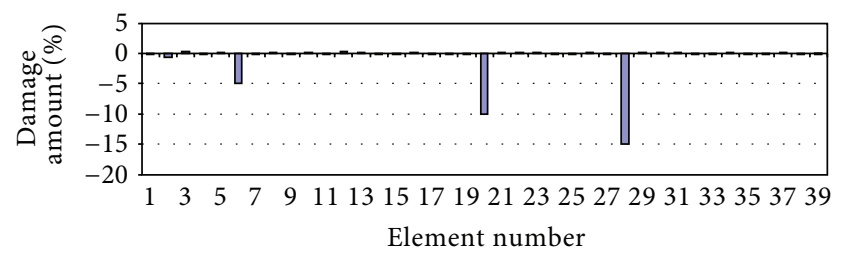

(b)

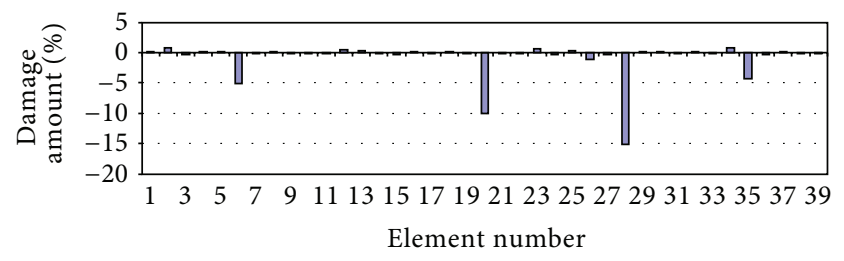

(c)

FIGURE 3: Inverse damage predictions for scenario 2 affected by the number of damaged-structure frequencies (NF), all original eigenvectors (NM) used, (a) 20 damaged-structure frequencies used, (b) 35 damaged-structure frequencies used, (c) 60 original eigenvectors (NM), and 35 damaged-structure frequencies (NF) used.

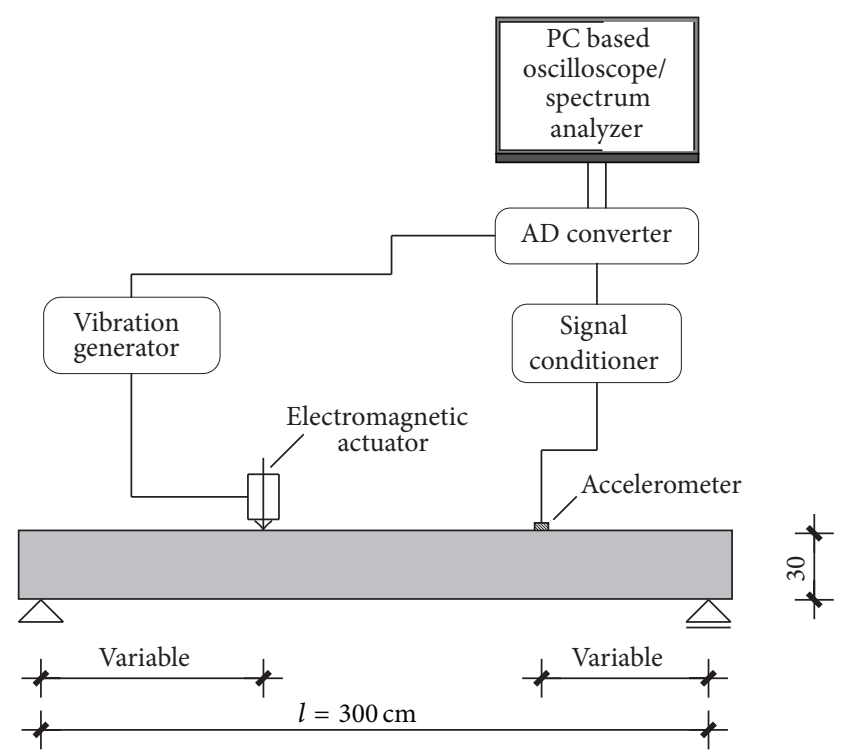

FIGURE 4: Experimental setup for natural frequency measurements.

The natural frequency measurements were made on reinforced concrete beam before and after damage. Damage was introduced incrementally by static loading to three levels.

Sinusoidal forced vibration tests were conducted on a simply supported reinforced concrete beam to obtain the frequencies. The vibration generator employed in the 


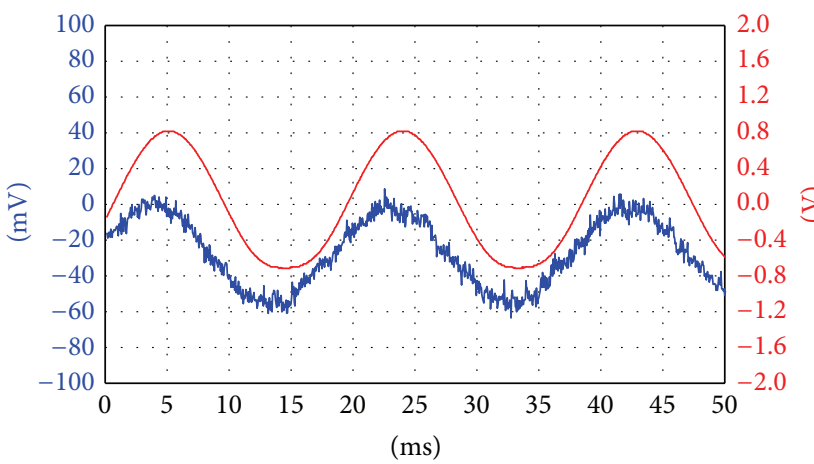

(a)

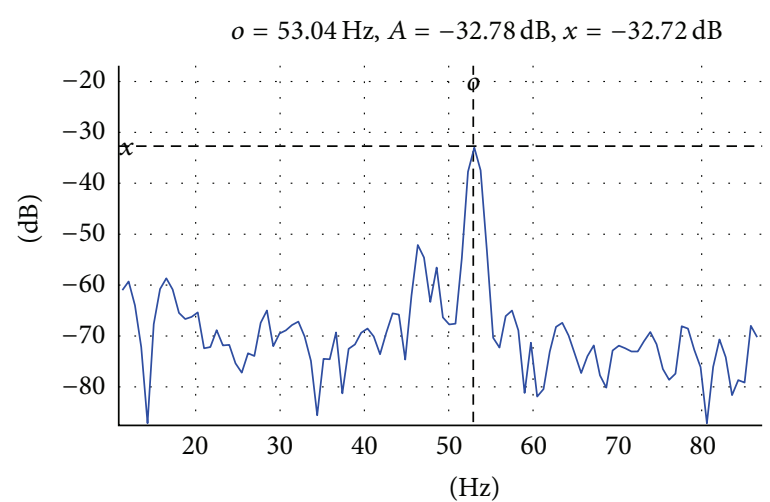

(b)

FIGURE 5: Typical structural acceleration responses: (a) time domain response and (b) spectrum of the time domain response.

vibration tests consisted of an electromagnetic actuator mounted vertically in a frame with steel plates attached to the free end of the rod of the actuator. The response of the structure at a point was measured using a piezoelectric accelerometer (Brüel\&Kjær-DeltaTron type 4508). The actuator and accelerometer were located at points of maximum deflection for the first eight bending modes. Both the excitation and response, after passing through a signal conditioner, were captured by an A/D converter (ADC-200/50). In the $\mathrm{A} / \mathrm{D}$ converter, these analog signals were first digitized and then processed further to a PC based oscilloscope/spectrum analyzer (PicoScope software). All data were recorded and analyzed using the PicoLog data acquisition software.

4.2. Experimental Results. Experimentally measured frequencies are obtained before and after each level of static concentrated load $(10,20$, and $30 \mathrm{kN})$ applied at the middle of the span of simply supported reinforced concrete beam.

Figure 5 shows a typical time domain response and the associated frequency spectrum for a reinforced concrete beam.

The experimental natural frequencies for the first eight bending modes for the laboratory model subjected to three levels of static load are given in Figure 6.

The estimated natural frequencies were then used in the direct iteration technique to determine the location and the extent of damage.

As expected, the development of cracks in the laboratory beam does cause decrease of stiffness and consequently decrease of structural natural frequencies.

4.3. Damage Identification. Based on the measured resonant frequencies for the undamaged and the damaged structures, a direct iteration technique was employed for structural damage identification.

The computer program used here for dynamic analysis and damage identification was developed for the solution of two-dimensional (2D) framed structures which have used beam-column elements. These elements each have six degrees of freedom, incorporating two translations and a rotation at

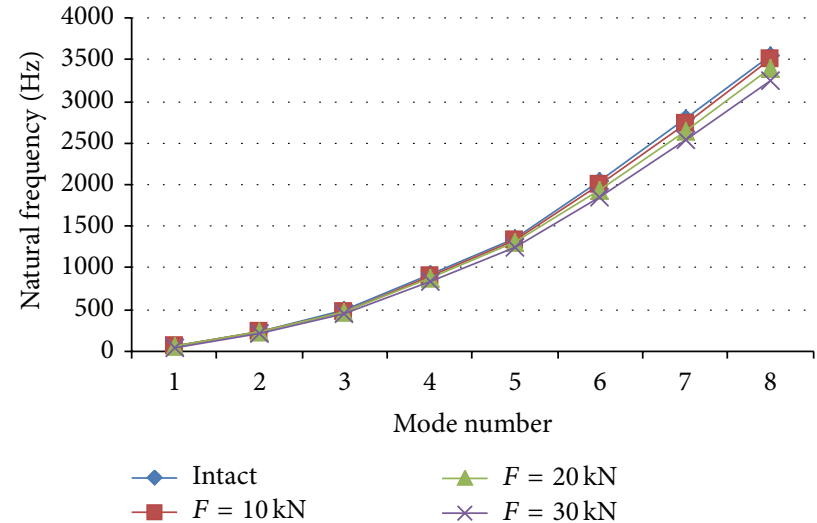

FIGURE 6: Experimental natural frequencies $(\mathrm{Hz})$ for the simply supported beam subjected to static loading.

\begin{tabular}{lc}
\multicolumn{1}{c}{ Parameters of the problem } \\
Total degrees of freedom & 18 \\
Finite-elements & 9 \\
Damage parameters & 9
\end{tabular}

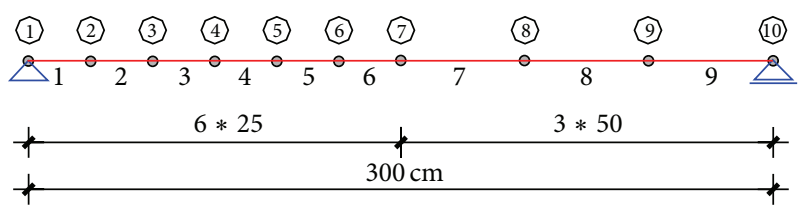

Figure 7: Beam model problem.

each node, although the axial displacement is ignored in this example.

In order to avoid problems associated with structural symmetry, 9 nonsymmetric finite-elements with 10 nodes and a total of 18 degrees of freedom are generated (Figure 7). All elements have the same material properties with experimentally estimated elastic modulus $E=3.4 \times 10^{7} \mathrm{kN} / \mathrm{m}^{2}$ and density $\rho=2.5 \mathrm{t} / \mathrm{m}^{3}$ and the same cross-sectional area $A=$ $0.06 \mathrm{~m}^{2}$ and second moment of area $I=4.8721 \times 10^{-4} \mathrm{~m}^{4}$. 


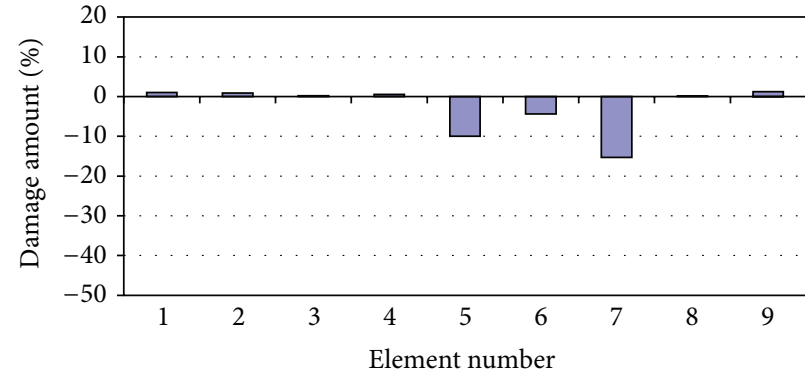

(a)

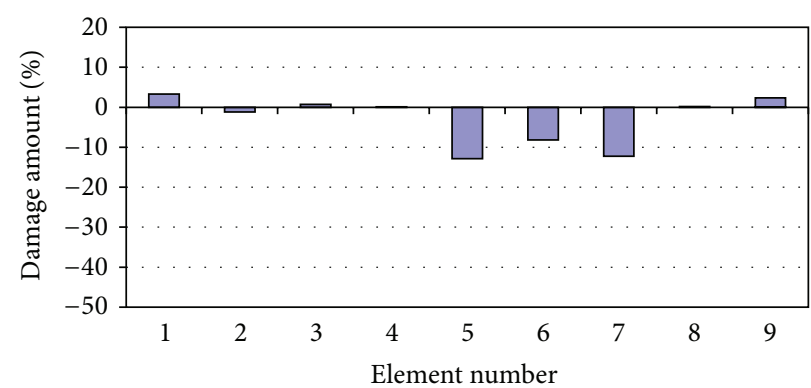

(b)

FIGURE 8: Inverse damage predictions for $F_{1}=10 \mathrm{kN}$, (a) 5 experimental frequencies for damaged structure used, and (b) 8 experimental frequencies for damaged structure used.

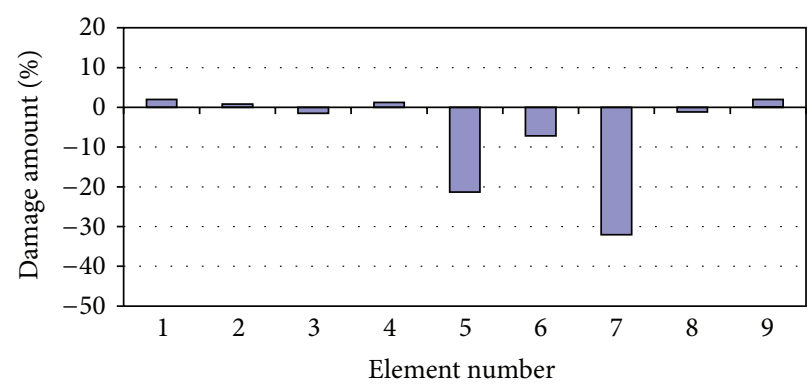

(a)

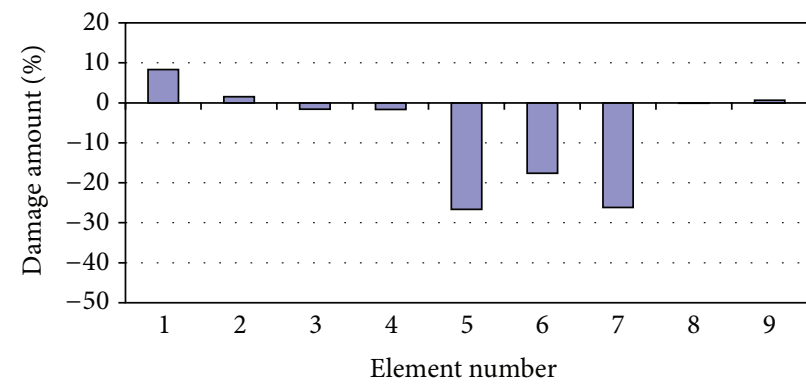

(b)

Figure 9: Inverse damage predictions for $F_{2}=20 \mathrm{kN}$, (a) 5 experimental frequencies for damaged structure used, and (b) 8 experimental frequencies for damaged structure used.

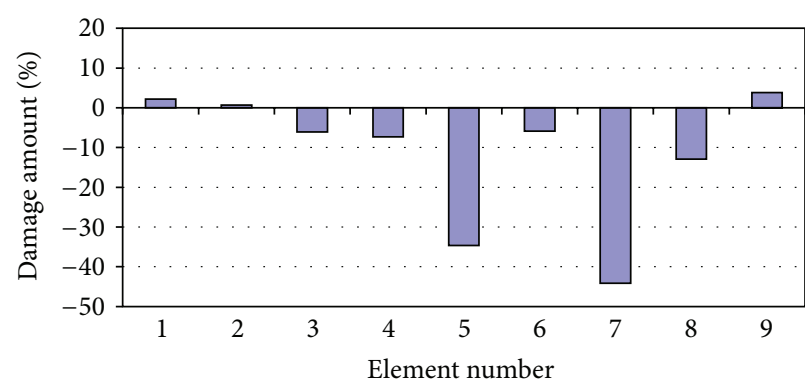

(a)

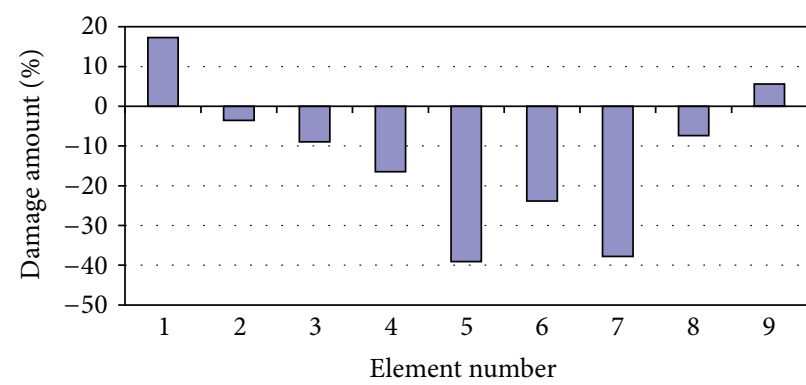

(b)

FiguRE 10: Inverse damage predictions for $F_{3}=30 \mathrm{kN}$, (a) 5 experimental frequencies for damaged structure used, and (b) 8 experimental frequencies for damaged structure used.

The geometry of the structure and element numbering are shown in Figure 7.

The results in Figures 8-10 are obtained from the inverse damage predictions from the direct iteration technique. All of the eigenvectors for the undamaged structure are considered in structural damage identification.

It is found that for a case with slight damage (due to static force of $10 \mathrm{kN}$ ) both the location and the amount of structural damage can be correctly estimated using only 5 experimental natural frequencies for the damaged structure (Figure 8(a)). For cases with more serious damage (due to static force of 20 and $30 \mathrm{kN}$ ) structural damage can be roughly predicted using only 5 experimental natural frequencies
(Figures 9(a) and 10(a)). However, if 8 experimental natural frequencies are used, both the location and the amount in either case can be determined correctly.

\section{Conclusions}

In this paper, damage identification method using the changes of measured natural frequencies has been applied experimentally. Results of the damage identification procedure are similar to those obtained from a numerical example and show that this method is capable of successfully identifying both the location and the extent of structural damage, including 
small and multiple damage. Several distinct advantages have been highlighted. Firstly, high-order (beyond the 4th order) natural frequencies for the damaged structure not more than NXE are required to identify structural damage. The predictions of structural damage improve with an increase of the number of natural frequencies for the damaged structure adopted. The less the structural damage is, the fewer natural frequencies are required. Secondly, no knowledge of mode shapes for the damaged structure is required since they can be obtained as a result of the proposed method. Thirdly, the method is suitable for symmetric structures, if a nonsymmetric element mesh is generated. Finally, the research results indicate that the ability to use high-frequency vibrational responses for the structural integrity assessment of real engineering structures such as bridges is an area that merits further investigation.

\section{Conflict of Interests}

The author declares that there is no conflict of interests regarding the publication of this paper.

\section{References}

[1] T. W. Lim and T. A. L. Kashangaki, "Structural damage detection of space truss structures using best achievable eigenvectors," AIAA Journal, vol. 32, no. 5, pp. 1049-1057, 1994.

[2] Z. Y. Shi, S. S. Law, and L. M. Zhang, "Damage localization by directly using incomplete mode shapes," Journal of Engineering Mechanics, vol. 126, no. 6, pp. 656-660, 2000.

[3] W.-X. Ren and G. de Roeck, "Structural damage identification using modal data. I: simulation verification," Journal of Structural Engineering, vol. 128, no. 1, pp. 87-95, 2002.

[4] J.-T. Kim, Y.-S. Ryu, H.-M. Cho, and N. Stubbs, "Damage identification in beam-type structures: frequency-based method vs mode-shape-based method," Engineering Structures, vol. 25, no. 1, pp. 57-67, 2003.

[5] H.-P. Chen, "Application of regularization methods to damage detection in large scale plane frame structures using incomplete noisy modal data," Engineering Structures, vol. 30, no. 11, pp. 3219-3227, 2008.

[6] P. Cawley and R. D. Adams, "The location of defects in structures from measurements of natural frequencies," Journal of Strain Analysis, vol. 14, no. 2, pp. 49-57, 1979.

[7] G. Hearn and R. B. Testa, "Modal analysis for damage detection in structures," Journal of Structural Engineering, vol. 117, no. 10, pp. 3042-3063, 1991.

[8] S. Hassiotis and G. D. Jeong, "Assessment of structural damage from natural frequency measurements," Computers and Structures, vol. 49, no. 4, pp. 679-691, 1993.

[9] N. Bicanic and H.-P. Chen, "Damage identification in framed structures using natural frequencies," International Journal for Numerical Methods in Engineering, vol. 40, no. 23, pp. 44514468, 1997.

[10] S. Hassiotis, "Identification of damage using natural frequencies and Markov parameters," Computers \& Structures, vol. 74, no. 3, pp. 365-373, 2000.

[11] C. R. Farrar and S. W. Doebling, "Damage detection II: field applications to large structures," in Modal Analysis and Testing, J. M. M. Silva and N. M. M. Maia, Eds., Nato Science Series,
Kluwer Academic Publishers, Dordrecht, The Netherlands, 1999.

[12] M. Raghavendrachar and A. Aktan, "Flexibility by multireference impact testing for bridge diagnostics," Journal of Structural Engineering, vol. 118, no. 8, pp. 2186-2203, 1992.

[13] C. R. Farrar and K. M. Cone, "Vibration testing of the I40 bridge before and after the introduction of damage," in Proceedings of the 13th International Modal Analysis Conference, pp. 203-209, Nashville, Tenn, USA, 1995.

[14] M. Cao, W. Xu, W. Ostachowicz, and Z. Su, "Damage identification for beams in noisy conditions based on Teager energy operator-wavelet transform modal curvature," Journal of Sound and Vibration, vol. 333, no. 6, pp. 1543-1553, 2014.

[15] N. M. M. Maia, "An introduction to the singular value decomposition technique (SVD)," in Proceedings of the 7th International Modal Analysis Conference, pp. 335-338, Las Vegas, Nev, USA, 1989.

[16] I. Mekjavić, Durability assessment of concrete bridges based on dynamic parameters [Ph.D. thesis], Faculty of Civil Engineering, University of Zagreb, Zagreb, Croatia, 2004.

[17] M. Biswas, A. K. Pandey, and M. M. Samman, "Diagnostic experimental spectral/modal analysis of a highway bridge," The International Journal of Analytical and Experimental Modal Analysis, vol. 5, no. 1, pp. 33-42, 1990.

[18] G. Feltrin and M. Motavalli, "Vibration-based damage detection on a highway bridge," in Proceedings of the 1st International Conference on Bridge Maintenance, Safety and Management (IABMAS '02), pp. 1-8, Barcelona, Spain, 2002.

[19] C. E. Ventura, R. Brincker, P. Andersen, and R. Cantieni, "Identification and damage detection studies of the Z24 highway bridge in Switzerland," in Proceedings of the 6th International Conference on Short and Medium Span Bridges, pp. 851-858, Vancouver, Canada, August 2002. 

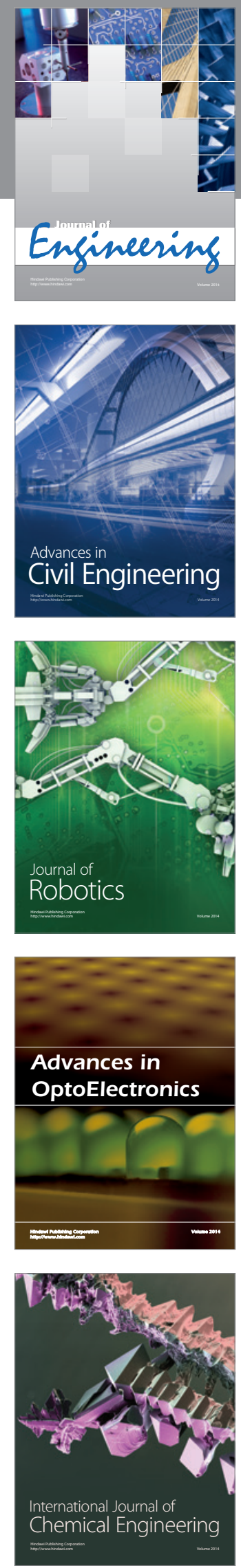

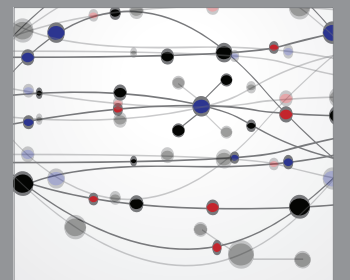

The Scientific World Journal
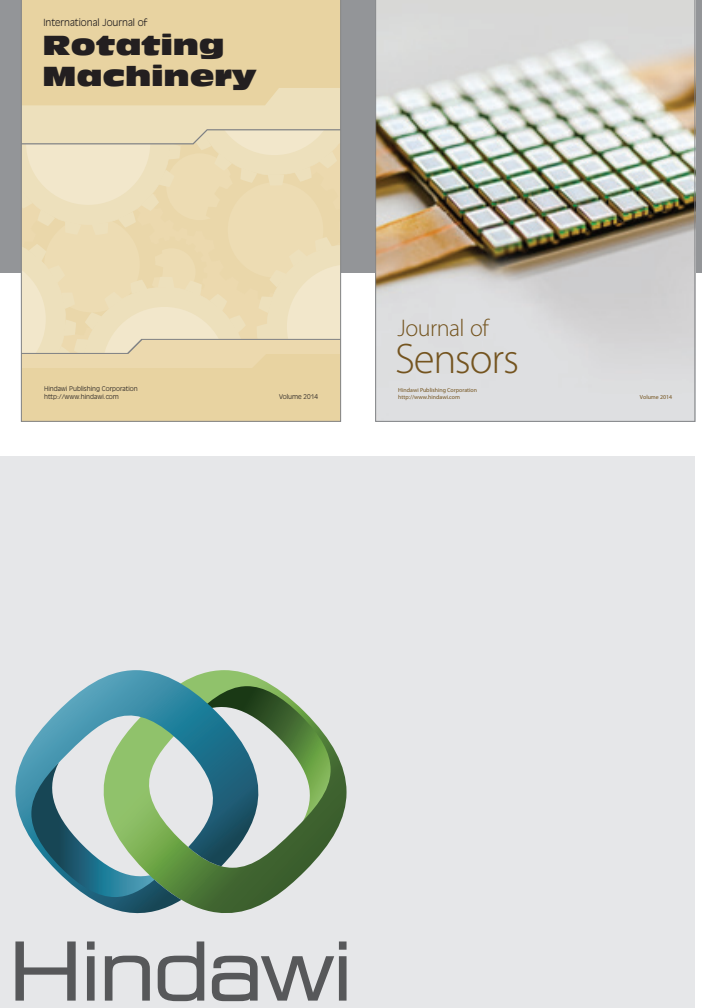

Submit your manuscripts at http://www.hindawi.com
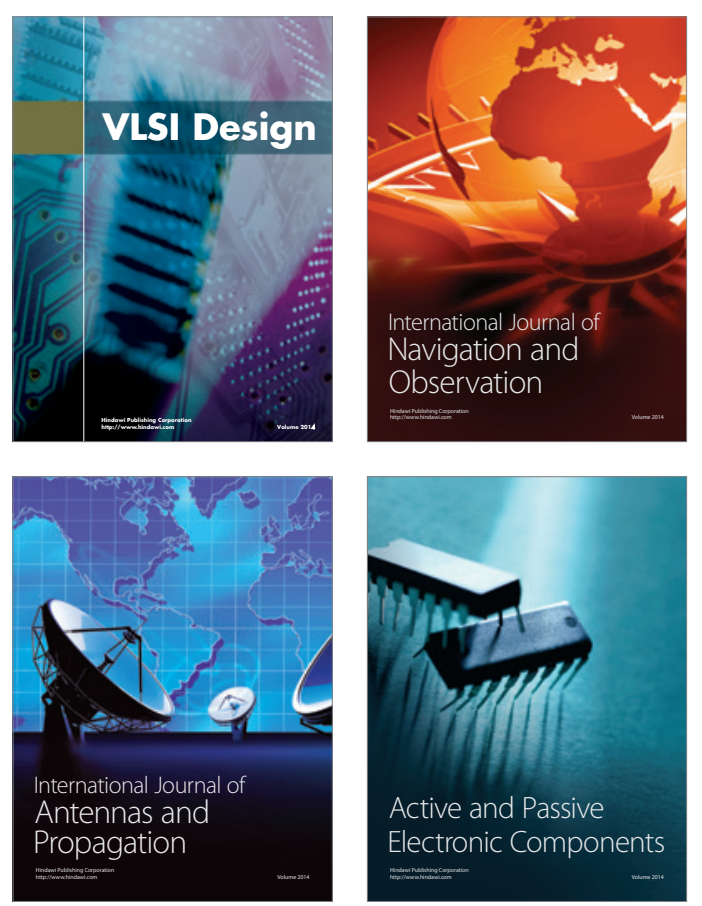
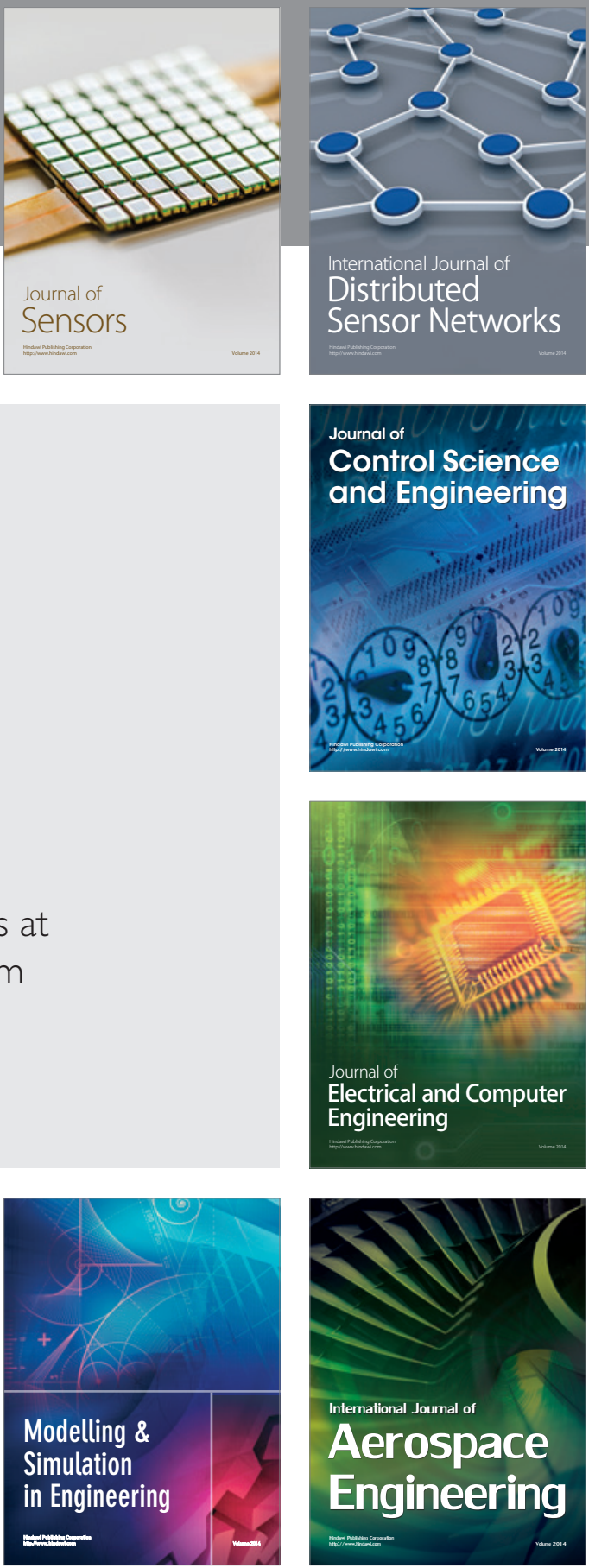

Journal of

Control Science

and Engineering
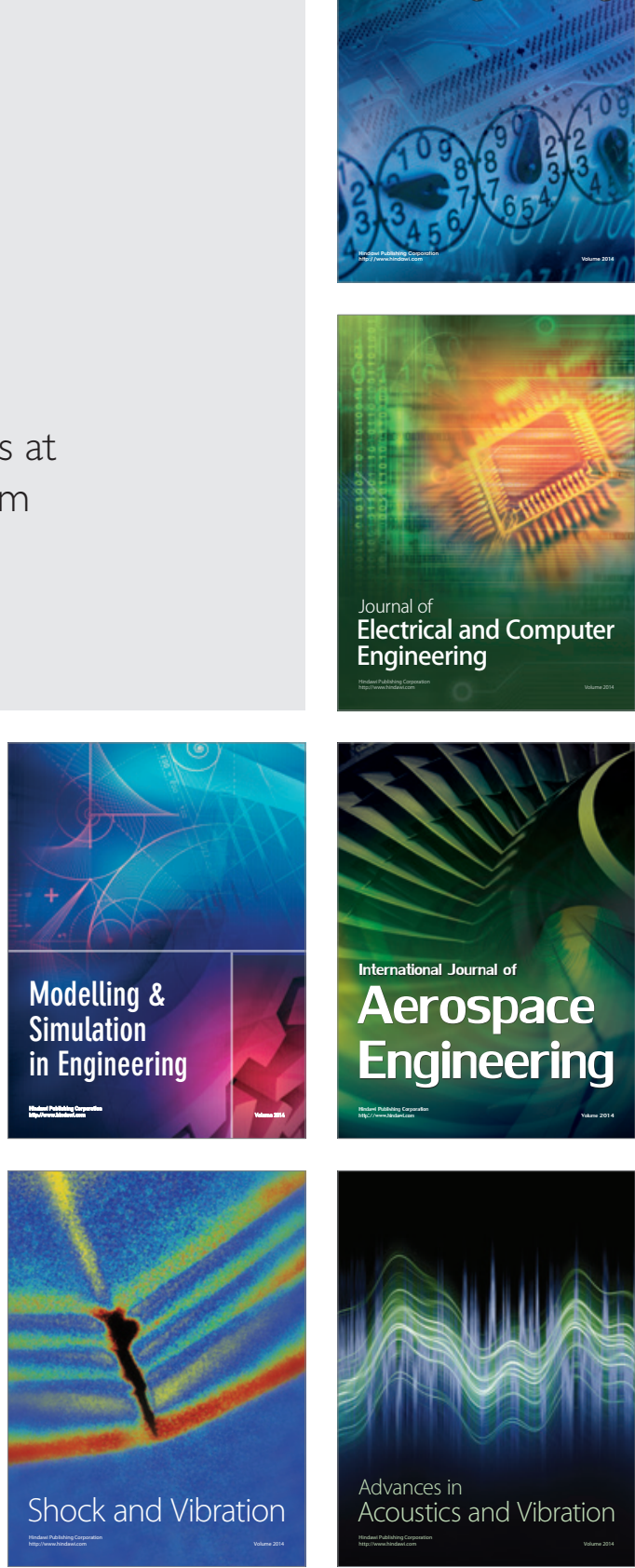